\title{
Surgical technique for revision surgery of cervical artificial disc replacements
}

\author{
Julia Onken, MD, ${ }^{1}$ Bernhard Meyer, MD, ${ }^{2}$ and Peter Vajkoczy, MD ${ }^{1}$ \\ 1Department of Neurosurgery, Charité Universitätsmedizin Berlin; and 2Department of Neurosurgery, TU Munich, Germany
}

OBJECTIVE Cervical artificial disc replacement (C-ADR) is a widely used procedure with low risk at implantation. Few cases have been reported about the surgical techniques of $C$-ADR revision. The authors describe their surgical experience with the explantation of a Galileo C-ADR.

METHODS Revision surgery was performed in a 58-year-old patient. Patient positioning and surgical opening techniques were performed as appropriate for anterior cervical decompression.

RESULTS Revision surgery via the initial anterior approach was successful following an atraumatic removal of the implant. Fusion of the C5-6 segment was performed without complications.

CONCLUSIONS In general, the authors observed recurrent nerve palsy and malpositioning of the revised implant in C-ADR revision surgery. Problems with implant removal did not occur because the fusion rate was low due to the short time between initial surgery and C-ADR revision surgery.

The video can be found here: https://youtu.be/32CUEDquinc.

KEYWORDS cervical artificial disc replacement; C-ADR; revision surgery; surgical technique; anterior cervical decompression; video 